
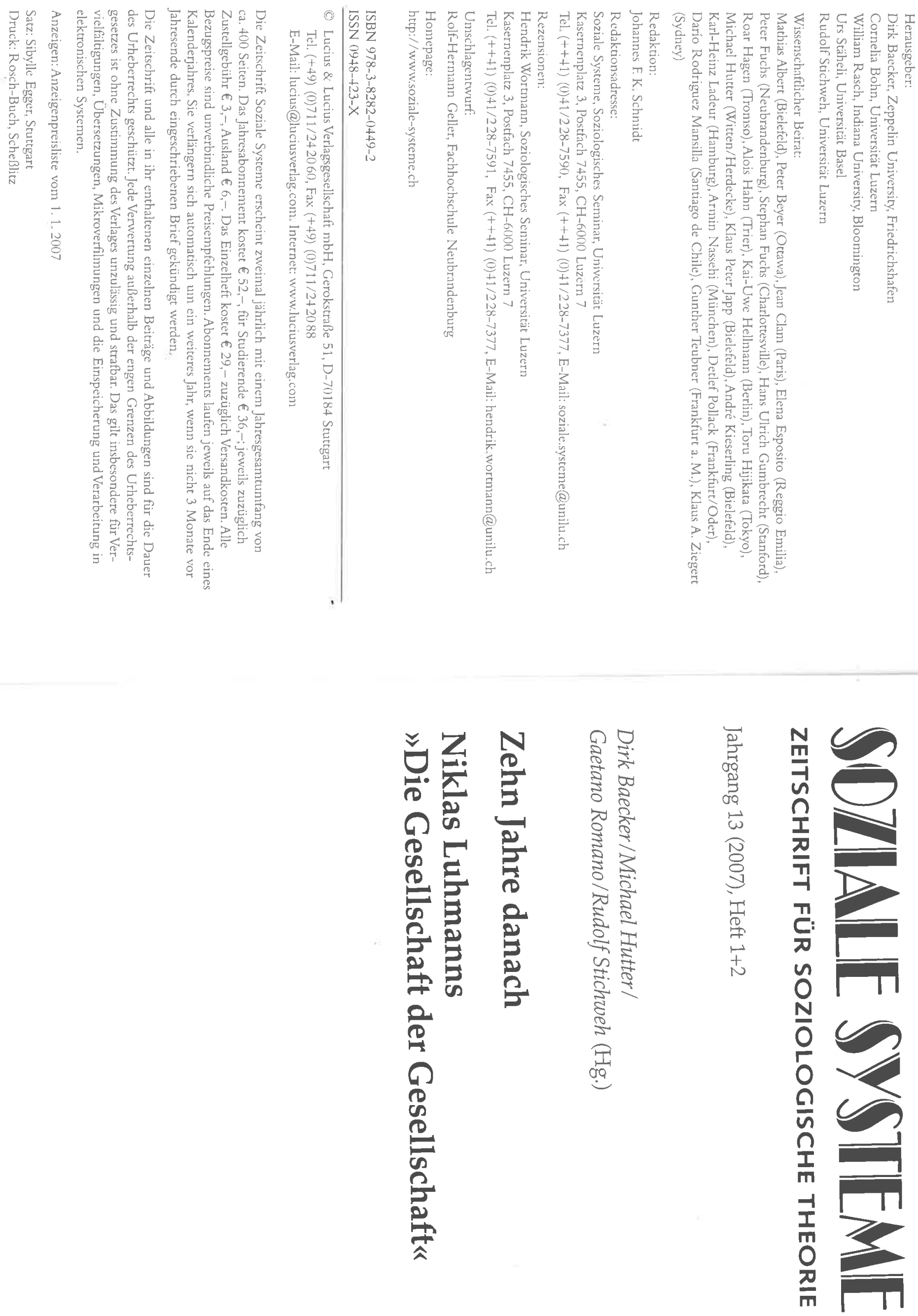
Alberto Cevolini

\section{Die Episodisierung der Gesellschaft}

Zusammenfassung: »Episodisierung « ist ein der wichtigsten aber zugleich bis heute am wenigstens erforschten Begriffe der Theorie gesellschaftlicher Differenzierung. Versucht man, den Episodisierungsbegriff schärfer zu bestimmen, versteht man, dass es nicht nur um die evolutiv hervorgegangene Differenz von Interaktion und Gesellschaft, sondern auch um die unwahrscheinliche Selbsterzeugung der Gesellschaft geht, obwohl die Gesamtgesellschaft nicht lediglich aus Interaktionen besteht. In diesem Beitrag wird die These untersucht, nach der eine Episodisierung der Gesellschaft in eigentlichem Sinne nur dann entsteht, wenn Interaktion und Gesellschaft sich voneinander so stark differenzieren, dass man nicht mehr voraussetzen kann, dass die Gesellschaft unmittelbar auf Interaktionen angewiesen ist. Von nun an bereitet man sich vor, Interaktionen in Form von Episoden und nicht mehr von ritualisierten Ereignissen zu organisieren.

I.

»Episodisierung « ist ein wichtiger aber zugleich bis heute sehr wenig erforschter Begriff der Gesellschaftstheorie. ${ }^{1}$ Versucht man, die soziologische Tragweite des dem Episodisierungsbegriff zugrunde liegenden Problembezugs schärfer zu bestimmen, versteht man, dass es nicht nur um die evolutiv hervorgegangene Differenz von Interaktion und Gesellschaft, sondern auch um die nach wie vor unwahrscheinliche Selbsterzeugung der Gesellschaft geht. Während man dennoch in Bezug auf die Interaktionssysteme bzw. die Gesamtgesellschaft schon seit langem auf eine große Anzahl von Forschungsarbeiten zurückgreifen kann, ist die Einheit der Differenz von Interaktion und Gesellschaft zutiefst vernachlässigt worden.

Diese Differenz stellt sich soziologisch als die ursprüngliche, nicht zu vermeidende Struktur der Gesellschaft dar (Luhmann 1997, 817). Einerseits wäre ohne Gesellschaft das Stattfinden eines Interaktionssystems nicht einmal möglich. Anfang und Ende einer Interaktion setzen immer das Dasein einer Gesellschaft voraus, die zweierlei strukturelle Vorbedingungen bietet, nämlich Sprachfähigkeiten, die die von der Gesellschaft selbst garantierte sinnhaft-selbstreferentielle Geschlossenheit der Kommunikation voraussetzen, und schnell wiedererkennbare Erwartungsstrukturen, die dazu dienen, die doppelte Kontingenz

1 Explizit in Zusammenhang damit steht nur der Artikel von Teubner (1987) zur Verfügung. 
jeweils zu organisieren und in anpassender Weise zu reduzieren, mit dem Ziel, Zeit und Aufmerksamkeit zu sparen und zu vermeiden, jedes Mal (den ganzen Prozess) wieder von vorne beginnen zu müssen (Luhmann 1984, 566ff.).

Andererseits beschränkt sich die Gesamtgesellschaft nicht einfach auf Interaktionen; sie besteht nicht einmal aus der Summe aller wirklich stattfindenden Interaktionen, obgleich in primitiven Gesellschaften wie z.B. den Stammesgesellschaften eben dies die übliche Vorstellung von »Gesellschaft« ist. Interaktionen kommen vielmehr als Episoden des Gesellschaftsvollzugs zustande, deren Anfang und Ende bestimmte Zäsuren in der Autopoiesis der Gesellschaft markieren (so Luhmann 1984, 553). ${ }^{2}$ In Hinsicht darauf fungiert die Gesellschaft als dasjenige System, das in sich selbst alles, was Kommunikation ist, einschließst, und sie bleibt zugleich als irritierende innergesellschaftliche Umwelt von Interaktionssystemen anwesend. In diesem Zusammenhang spricht Luhmann von »Doppelzugriff«. Das bedeutet, dass jede Interaktion, da sie einfach Kommunikation zustande bringt, Gesellschaft vollzieht, während die Gesellschaft sich gleichzeitig als Umwelt der Interaktion in vielfältiger Weise bemerkbar macht (Luhmann 1997, 817). Die Gesamtgesellschaft ist also, da sie zugleich als System und Umwelt im Interaktionsverlauf vorkommt, durch eine auffällige Paradoxie gekennzeichnet: Sie ist kurz ausgedrückt das Ökosystem der Interaktionen (Luhmann 1984, 589).

Der Doppelzugriff kann in alle drei Sinndimensionen der Kommunikation aufgegliedert werden: In der Sozialdimension berücksichtigt man in einem bestimmten Ausmaß, welches von der Evolution der jeweiligen Gesellschaft abhängt, andere eigene Rollen, nämlich die Rollen, die die Teilnehmer der Interaktion außerhalb derselben Interaktion, und zwar in unterschiedlichen sozialen Kontexten, möglicherweise einnehmen könnten. In der Sachdimension wird es vor allem möglich, die Unterscheidung von anwesend und abwesend als anwesend zu behandeln, indem man z.B. über Abwesende(s) bzw. über Themen, die man aufgrund der öffentlichen Meinung (früher stattdessen: aufgrund der Rhetorik) voraussetzen kann, spricht. In der Zeitdimension handelt es sich um die aus der Gesellschaft hervorgegangene Möglichkeit der Episodenbildung.

In Anschluss daran vollzieht die Gesellschaft eine Doppelleistung: Einerseits fungiert die Gesellschaft während des Verlaufs einer Interaktion als ihre betreffende innergesellschaftliche Umwelt und dies gewährleistet nicht zuletzt symbolisch, dass alles, was geschieht, gleichzeitig geschieht, ohne dass irgendetwas bzw. irgendjemand in die Zukunft vorauseilen oder in der Vergangenheit zurückbleiben kann. Die Gesellschaft stellt also die Synchronizität von allem sicher, was im System bzw. in der Umwelt stattfindet. Andererseits ermöglicht die Gesellschaft, indem sie sich als Episode mit einem eigenen Anfang und

2 Anfang und Ende wären in diesem Sinne ein »Artefakt« der Autopoiesis des Systems (Luhmann 1997, 816). 
einem eigenen Ende realisiert, die Bildung von Diachronizitäten, die sich unter der Voraussetzung entfalten, dass keine Interaktion mit der Notwendigkeit belastet ist, das vollständige Kommunikationsvermögen zum letzten Mal zu reproduzieren (Luhmann 1990d, 94; 1997, 819). Zusammenfassend: Gesellschaft gibt es immer vor, während und nach der Interaktion und dies ermöglicht die Gestaltung von Episoden unter der Bedingung der Nichtepisodisierbarkeit der Gesamtgesellschaft.

\section{II.}

Setzen wir diesen allgemeinen Ansatz voraus, neigen wir folglich zu einer schärferen Bestimmung des Episodenbegriffs und halten diese Abgrenzung für unentbehrlich, will man die Episodisierung der Gesellschaft als ein Ergebnis der gesellschaftlichen Evolution auffassen.

Wenn im Folgenden von »Episode« die Rede ist, dann ist immer eine besondere Art von Interaktion gemeint. Wie jede Interaktion lässt sich auch eine Episode nur dann bilden, wenn zumindest zwei Beteiligte (z.B. Arzt/Patient) gleichzeitig anwesend sind und sich einander reflexiv wahrnehmen - d.h. sie nehmen wahr und nehmen infolgedessen auch das Wahrnehmenkönnen des Anderen und das jeweils eigene Wahrgenommenwerden wahr. Nicht alle Interaktionen sind jedoch im strikten Sinne Episoden. Damit man von Episoden reden kann, müssen die Interaktionen in dem Erfüllen sozialer Funktionen verankert werden. Das Interaktionssystem dient in diesem Falle dazu, eine selbsterzeugte Unsicherheit zu entfalten, die im Verlaufe der Interaktion selbst oder in langfristig geplanten Sequenzen von Episoden, die man Perioden nennen darf, wie etwa im Falle einer Verhandlung im Gericht oder der Schulzeit, über die Bildung von Rollenasymmetrisierungen (Richter/Beklagte, Schüler/Lehrer usw.) absorbiert wird. Diese Rollenasymmetrien ermöglichen eine rekursive Gliederung der Kommunikation, indem sie aufgrund der jeweils entsprechenden Funktion codierte Beobachtungen zweiter Ordnung im Hinblick auf eine Entscheidung in Gang setzen, die in einem über Alternativen wie z.B. Verurteilung/Freispruch, Erfolg/Misserfolg eröffneten Spielraum von Kontingenz getroffen werden muss.

Wie alle Interaktionen sind auch Episoden außerdem mit einem Anfang und einem Ende ausgestattet, denn ihre Bildung muss zweierlei Vorproblemen lösen: wie man anfängt und wie/wann man aufhört? Die Antwort auf diese Fragen hängt von der Form der Differenzierung sozialer Strukturen und von dem Kommunikationsvermögen der Medien ab. Das schließt natürlich nicht aus, dass der Episodenbildung bestimmte Umweltbedingungen zugrunde liegen: Die Körper ermüden, bekommen Hunger, das Bewusstsein wird abgelenkt, langweilt sich: man gähnt und man deutet an, dass es besser wäre, wenn man mit dem Kommunizieren aufhören würde. Anders gesagt: Die Episoden, wie 
im übrigen alle Interaktionen, nehmen Zeit und Aufmerksamkeit in Anspruch - und in beiden Fällen handelt es sich um knappe Ressourcen. Man muss also das Sichmiteinanderunterhalten unterbrechen und das Wiederzusammentreffen planen.

Noch wichtiger scheinen allerdings die Systembedingungen zu sein: Die Frage bezieht sich in diesem Fall nicht lediglich darauf, wie man von einem Anfang zu einem Ende, sondern vielmehr wie man von einem Ende zu einem neuen (d.h. späteren) Anfang übergehen kann. Dieser Übergang muss nach wie vor festgelegt werden, bevor man anfängt, oder wenigstens bevor man zum Ende kommt, wenn man vermeiden will, dass die Episodenbildung zu einer bloßen Episodizität wird. Es geht im Wesentlichen um die operative Fähigkeit, eine strukturelle Episodenverknüpfung unter der zentralen Voraussetzung sicherzustellen, dass die Episodenverknüpfung selbst keine Episode ist (und nicht einmal sein darf). Episoden sind in diesem Sinne immer mit dem Zweck eingerichtet, das Verzichten auf das Wiederzusammentreffen zu verschieben ${ }^{3}$ - eben das, was im Gegenteil in üblichen Interaktionen normalerweise passiert: Es reicht dazu aus, Generalisierungen wie z.B. »Bis zum nächsten Mal« auszunutzen.

Die Episoden, ebenso wie die Ereignisse, werden nicht von Strukturen geschaffen, sondern von anderen Episoden aufgrund von Strukturen, die von der Gesellschaft jeweils zurVerfügung gestellt werden. Das System führt seine eigene Rekursivität auf der Ebene der stets immanenten Vorläufigkeit von Ereignissen und betreibt auf diese Weise eine für das Bilden eines eigenen Gedächtnisses unentbehrliche Reduzierung von Komplexität. Diese Fähigkeit der Gesellschaft, Episoden mit weiteren Episoden gleicher Art nicht zufällig sondern rekursiv zu verknüpfen, hängt von der Form der Selbstorganisation des Gesamtsystems ab. Der Umwelt kommt ihrerseits immer lediglich eine destruktive Rolle zu: Der Lehrer ist krank, der Unterricht fällt aus. Die Struktur ist in diesem Sinne die Voraussetzung und zugleich das Ergebnis von Bedürfnissen nach Koordination auf der Ebene von sozialen und sachlichen Verhältnissen zwischen System- und Umweltereignissen (oder kürzer gesagt: von Bedürfnissen nach Synchronisation), die über Zeitbindungen gelöst werden können.

\section{III.}

Jedes Sozialsystem vergeht nicht nur mit der Zeit, sondern nimmt auch die Zeit in Anspruch, die es hat, nämlich die Zeit, die das System sich selbst zurVerfügung stellt, um Kommunikation an weitere Kommunikation anzuschließen. So wie alle operativ geschlossenen Systeme, erreicht auch die Gesellschaft eine

3 Dazu muss man aber motivieren und das Problem betrifft genau die Frage: Wie? Vgl. auch Luhmann 1984, 566. 
Mindestkomplexität, wenn sie auf eins-zu-eins-Kopplungen von System- und Umweltereignissen verzichten und den Mangel an operativer Anschlussfähigkeit mit der äußeren Umwelt über interne Einrichtungen - insbesondere in diesem Falle: über zeitliche Einrichtungen - aufheben kann (Luhmann 1997, 83). Auf diese Weise entsteht innerhalb des Systems eine Eigenzeit, die sich von der Weltzeit (d.h. von der Zeit der Welt) abkoppeln kann, obwohl beide miteinander koordiniert bleiben. Man besucht die Vorlesung, auch wenn es regnet. Aufgrund dieser zeitlichen Entkopplung gewinnt das System an Leistungsfähigkeit, die eine wichtige Rolle für die Fortsetzung seiner Operationen spielt; es kann z.B. Beschleunigungen und Verzögerungen betreiben, erinnern und planen, kurz: Zeit gewinnen und Zeit verwalten.

Das ist aber nur dann möglich, wenn das System sich selbst beobachten kann, indem es die Wiedereinführung der Unterscheidung System/Umwelt in das unterscheidende System selbst vollzieht. Das System betrachtet sich, als wäre es ein äußerer Beobachter. Man muss dazu Externalisierungen einrichten, wie die Perspektive eines Alter-Egos in der Sozialdimension, oder künstliche Behelfe (etwa die Uhr bzw. den Kalender) in der Zeitdimension (Luhmann 1990a, 20). Diese Externalisierungen werden in jedem Fall immer intern eingerichtet - sonst wären sie für das betreffende System unzugänglich. Denn keine Gesellschaft kann auf generalisierte, intersubjektiv begreifbare Mittel für die Bezeichnung und Messung der Zeit verzichten, insbesondere wenn es darum geht, das Anwesendsein unterschiedlicher Leute, deren Aktivitäten undVerhalten koordiniert werden müssen, zu gleichen Zeitpunkten zu gewährleisten. ${ }^{4}$ In Stammesgesellschaften ist es besonders schwierig, eine deutliche Differenzierung zwischen Interaktion und Gesellschaft treffen zu können und es ist außerdem kaum vorstellbar, dass die Kommunikation anders ablaufen kann, wenn nicht interaktiv in Form einer mündlichen Kommunikation. Hier wirkt das Wahrnehmen als Medium der Synchronisation und die Sozialität wird in jedem Fall als Interaktion unter Anwesenden aufgefasst. ${ }^{5}$ Elemente des medialen Substrats sind in diesem Fall die Wahrnehmungsfähigkeiten, deren strikte Kopplung das Einprägen von vielerlei Formen des koordinierten Verhaltens ermöglicht. Die Zeit stellt hier keine wirklich autonome Sinndimension der Kommunikation dar. Es ist nicht die Zeit, die die Tätigkeiten misst, sondern es sind die Tätigkeiten, die die Zeit messen. In diesen Gesellschaften sind die Kalender im wesentlichen phänomenale Kalender (Mbiti 1969, 19): Die Sachdimension hat den Primat für die Einstellung der Kommunikation inne, denn man zählt anhand von auf Redundanz basierten Ereignissen (Mondmonate der Schwangerschaft; notwendige Reisetage, um ein bestimmtes Ziel zu erreichen, usw.), während das Betonen der Zeit die gesellschaftlichen Tätigkeiten

4 Sieht man den klassischen Beitrag von Sorokin / Merton 1937.

5 Vgl. Luckmann 1991, 156 und Luhmann 1990d, 109-113. Luhmann spricht in diesem Fall von »Nahsynchronisation« (insb. 110), Schorr $(1990,118)$ von »Kooperation«, Giddens (1990) von »sozialer Integration «. 
wider spiegelt, die grundsätzlich entweder heilig oder profan sein können (Sorokin/Merton 1937, 620f.). In beiden Fällen wird jedes Mal, wenn es sich um Interaktionen handelt, bei denen eine soziale Funktion eine Rolle spielt, die Zulassung zur Teilnahme über Ritualisierung reguliert.

Der Ritus dient in allen diesen Fällen als Inszenierung für das Reaktivieren von Selbstbeschreibungen, die das soziale Gedächtnis enthalten. Wer an dem Ritus teilnimmt, wird in der Tat in eine besondere Zäsur zwischen heiliger und profaner Zeit verwickelt: Der Ritus nimmt Zeit in Anspruch, verbraucht irreversible Zeit, aber ermöglicht den Beteiligten, zugleich an einer außerzeitlichen reversiblen Dimension teilzunehmen, der der Ewigkeit, wo die erinnerungswürdigen Inhalte des sozialen Wissens re-aktualisiert werden (Havelock 1986; Eliade 1984; Cazeneuve 1996). Die Ritualisierung wirkt in diesem Sinne als ein Gedächtnis zweiter Ebene: Man darf nicht vergessen, sich zu erinnern. Dies ist aber noch nicht ausreichend, um die Anwesenheit der Teilnehmer sicher zu stellen: Menschen werden krank und sterben. Um religiöse Feste, Zeremonien, Riten der Initiation, Gedächtnisfeiern usw., welche eventuell die Anwesenheit von Personen, die weit voneinander entfernt leben, erfordert, so organisieren zu können, dass das Ende des Rituals nicht das Ende der Möglichkeit einer erneuten Interaktion bedeutet, ist es notwendig, sich Kriterien zu bedienen, die über die Vorläufigkeit von Körpern und Bewusstseinszuständen hinausgehen: Man kann hier zum Beispiel das Ritual an dem zyklischen Verlauf der Weltzeit ankoppeln, deren Rundlauf die Wiederholbarkeit des Sichwiedertreffens garantiert (Luhmann 1990d, 104). Die Gesellschaft verabredet sich, wenn man so sagen darf, und erreicht auf diese Weise eine dynamische Stabilität in der Zeitdimension, indem sie bestimmte Orientierungen an externen Periodizitäten in Anspruch nimmt und aufgrund dessen liturgische Kalender bildet.

\section{IV.}

Aus der fortschreitenden Verstädterung und der späteren Durchsetzung einer stratifizierten Gesellschaft ergeben sich wichtige Konsequenzen, vor allem in Bezug auf die soziale Zeitlichkeit. Die Hypothese lautet, dass im Gegensatz zu der Unterschicht, die weiterhin im Wesentlichen in typischen Formen der Segmentierung lebt, die Oberschichten zeitliche Einrichtungen unterschiedlicher Art zu erfahren beginnen. Während das Volk mit der zyklischen Zeit der Natur verbunden bleibt, welche außerdem die liturgische Zeit des Heiligen symbolisch markiert und von der aus es möglich wird, den Spielraum des Profanen selbst abzugrenzen, löst sich der Adel relativ betrachtet von diesen Sachbindungen los und fängt an, abstraktere Koordinationsmöglichkeiten zu betreiben.

Dies kann in allen korporativen Genossenschaften festgestellt werden, wie Zünfte, Universitäten und Kloster. Es wird im übrigen eben in den Klöstern 
um das 13. Jahrhundert herum die Schlaguhr erfunden, während Mitte des 14. Jahrhunderts die ersten öffentlichen Uhren erscheinen, die symbolischer Weise auf Kirchtürmen oder auf den höchsten Türmen der Stadt angebracht werden (Cipolla 1981).Vom Beginn des 15. Jahrhunderts an findet man immer häufiger Uhrzeitangaben in städtischen Statuten, in Rats- und Gerichtsverordnungen, in Polizeireglements und natürlich auch in Schulordnungen und in den Satzungen der Universitäten (Dohrn-van Rossum 1989, 108ff.). Das trägt dazu bei, dass eine relative Entkopplung von Eigenzeiten in Gang gesetzt wird; der Unterricht wird z.B. abstrakt befristet und es wird von nun an immer deutlicher, dass die Dauer (eines Lehrvortrags, einer Predigt) unabhängig von dem entsprechenden Inhalt ist und dass man infolgedessen der vorgesehenen Frist schon bei der Vorbereitung eines Referats Rechnung tragen muss (Dohrn-van Rossum 1989, 111).

Die Uhren werden andererseits vor allem als Wecker für das Gebet in Klöstern verwendet, wobei die Dauer von dem Inhalt der Gebete festgesetzt wird, die immer in der gleichen Reihenfolge zu verrichten waren (Dohrn-van Rossum 1989, 101). ${ }^{6}$ Es ist allerdings zu bedenken, dass dieser allgemeine Synchronisationsbedarf nur innerhalb der jeweiligen Korporation (des Klosters, der Universität) an einer bestimmten Bedeutung gewann, während die Koordinationslösungen nicht dazu erdacht waren, um unmittelbar vom Kloster in die Gesellschaft übertragen zu werden. In gleicher Weise dient die Uhr, sobald sie auf der Turmuhr erscheint, dazu, die Aktivitäten der Stadt und nicht die des Landes zu koordinieren. Die Zeitbezeichnungen waren außerdem ganz ungenau: Die Turmuhr diente dazu, »die Stunden anzuzeigen«, und nicht dazu, die Zeit chronometrisch zu messen. Statt einer exakten Messung der Zeit wird noch die Bestimmung einer Zeitqualität bevorzugt: Die Abschnitte, in die die Zeit sich gliedert, werden durch ein Adjektiv gekennzeichnet und man spricht jeweils von tempora prisca, tempora aurea, nostra infelicia tempora usw. (Leclercq 1974, 3f.). Im Mittelalter wurde also die Uhr eher dazu verwendet, der profanen Zeit eine religiöse Prägung zu verleihen, was im übrigen vom metaphorischen Gebrauch der Uhr (horologium) als Symbol für das Betonen des liturgischen Rhythmus des Alltagslebens bewiesen wird (Dante, Paradiso, X, 10, 139ff.) - ebenso galten die astronomischen Zeitvorstellungen der Hochkulturen im wesentlichen als symbolische Widerspiegelung der kosmischen Ordnung religiöser Art, welche die Selbstrechtfertigung der Gesellschaft beinhaltete.

\section{V.}

Die übliche Vorstellung, die Erfindung der Schlaguhr habe eine Rationalisierung und eine entsprechende »Entzauberung « der modernen Zeitlichkeit aus-

6 In Bezug auf dasselbe Problem vgl. auch Zerubavel 1985, 69. 
gelöst, hält nicht nur den Ergebnissen einer systemtheoretischen Forschung nicht stand, sondern muss sogar umgekehrt werden (Mumford 2005, Kap. I). Zum einen kann man nicht erklären, warum die mechanische Uhr, die bereits im 13. Jahrhundert existierte, erst im 17./18. Jahrhundert auf der Ebene der Gesamtgesellschaft zu einem Synchronisationsmittel wird; zum anderen behandelt man einen Umweltfaktor als Ursache für die Umstrukturierung des Systems. Die Faktoren der Entwicklung müssen hingegen innerhalb des Systems selbst gesucht werden.

Wir nehmen zunächst an, dass die Erfindung des Buchdrucks in dieser Hinsicht eine zentrale Rolle in dem Sinne spielt, dass sie Interaktion und Gesellschaft voneinander relativ »abhakt « und die Differenz von diesen unterschiedlichen Sozialsystemen verstärkt. Von nun an wird es deutlich, dass Kommunikation ablaufen kann, ohne dass man an besonderen Interaktionen teilnehmen muss. Man lernt »in Stille und in Einsamkeit« (Condorcet 1989, 227), man orientiert sich in der Gesellschaft aufgrund einer anonymen, unpersönlichen Kommunikation, die sich aufgrund vorangegangener anonymer, unpersönlicher Kommunikation durch intern eingerichtete Bedingungen reproduziert, die von sozialen Umständen und vor allem von Rangdifferenzen absehen. Je unabhängiger die Gesellschaft von den einzelnen Interaktionen wird, desto unabhängiger werden die einzelnen Interaktionen von der Gesellschaft mit dem Vorteil, dass sie die Gesamtgesellschaft nicht mehr reproduzieren und multifunktionale Aufgaben erfüllen müssen (Luhmann 1987, 115ff.).

Andererseits trägt die Umformung der Differenzierung sozialer Strukturen zur Modernisierung der Gesellschaft bei. Der zunehmende Abbau der Stratifikation und die entsprechende Durchsetzung der funktionalen Differenzierung lässt den Primat der Sozialdimension für die Zulassung zur Teilnahme an den auf soziale Funktionen angewiesenen Interaktionsystemen schwinden. Wenn früher die Rangdifferenzen durch die Stilisierung und die zeremonielle Markierung der Formen des Unterhaltens zugleich kondensiert und konfirmiert wurden (Luhmann 1997, 861), ${ }^{7}$ geht man von nun an von einem spezifischen Universalismus aus, aufgrund dessen jedermann unabhängig von seiner Herkunft in den Genuss von Erziehung kommt; alle haben das Anrecht auf Rechtsschutz; jeder kann von einer Organisation eingestellt werden, vorausgesetzt, er hat die notwendigen Kompetenzen; und jeder kann Zahlungen leisten, vorausgesetzt, er verfügt über Geld. Luhmann spricht in diesem Zusammenhang, insbesondere in Bezug auf das Teilnehmendürfen an Episoden im Erziehungssystem, von »Homogenisierung « des Anfangs (Luhmann 1990b, insbes. 90.). Infolgedessen geht der Primat in der Autopoiesis der Gesellschaft von der Sozialdimension, welche grundsätzlich die Freiheitsgrade in der Sachdimension der Kommunikation beschränkte, zur Zeitdimension über, welche zur Annahme der Sozialbindungen zwingt, die von den Rollenasymmetrisierungen

7 »Kondensiert« und »konfirmiert« im Sinne der Spencer Browns (1979) Logik der Form. 
durchgesetzt werden, welche dem jeweiligen funktional differenzierten Teilsystem, in dem sich die Episoden von Interaktion realisieren, entsprechen. Die Zeitdimension erweist sich übrigens gegenüber der Sachdimension der Kommunikation als völlig gleichgültig: Dass der Unterricht um 10 Uhr anfängt, verspricht noch nicht, dass er interessant sein wird. Man geht von einer sozialen $\mathrm{zu}$ einer systemischen Integration (Giddens) über, d.h. die Integration wird über Zeitdispositionen gewährleistet und dient dazu, Zeitbindungen in Freiheitsgrade zu transformieren (so Luhmann 1984, 579). ${ }^{8}$

Die zunehmende Distanzierung von Interaktion und Gesellschaft erzeugt ferner einen Synchronisationsbedarf, welcher nicht mehr unter der Voraussetzung erfüllt werden kann, dass im Allgemeinen alle die gleichen sozialen und sachlichen Kontexte teilen. Man muss nun eher von der gegenteiligen Annahme ausgehen und ein im Vergleich zur Wahrnehmung abstrakteres Medium suchen. Gerade jetzt kann die Zeit als Medium der Synchronisation in Anspruch genommen werden, indem man ihr Abstraktions- und Homogenisierungsvermögen ausnützt. Es geht in diesem Falle nicht mehr um das Anwesendseinkönnen der Beteiligten, das in verschiedenen Formen des Verhaltens kombiniert werden kann, sondern eher um Jetztpunkte, die in allerhand möglichen Formen von Fristen und Terminen unter der Bedingung der Gleichzeitigkeit von allem, was geschieht, strikt gekoppelt werden können (Luhmann 1990d, 116). Diese Homogenisierung ermöglicht eine zunehmende Komplexität in dem Maße, wie das Befristetwerden mit unterschiedlichen sozialen Perspektiven verbunden wird: Während früher die Aktivitäten in linearer Weise verliefen, ist es nun möglich, unterschiedlichen Aktivitäten, die zueinander gesehen parallel verlaufen, zu synchronisieren. Man geht von der Zeit der Natur zu einer »Logistik der Zeit« über (Luhmann 1984, 256; vgl. auch Luckmann 1991, 157ff.). ${ }^{9}$ Die Uhr und der Kalender, verstanden als Mittel der Messung, die auf einer linearen und chronometrischen Konzeption der Zeit basieren, sind der offensichtliche Beweis für die Tatsache, dass die Zeit eine wirklich autonome Sinndimension ist: wenn früher Uhr und Kalender dazu dienten, Redundanzen zu produzieren, dienen sie nun eher dazu, Varietät unter der Bedingung einer allgemeinen zeitlichen Koordination zu ermöglichen. ${ }^{10}$ Von nun an wird die Kohärenz nicht mehr in dem zyklischen Ablauf der Natur und zugleich in der Reversibilität der mythischen Zeit, sondern vielmehr in der Irreversibilität der chronometrischen Zeit gefunden. Die Komplexität von sozialen Systemen wird temporalisiert und man entwickelt entsprechend eine Präferenz für zeitlimitierte Ordnungen, die jeweils die Form einer Periode bzw.

8 Giddens $(1990,135)$ behauptet, dass die Zeit in die »kalkulierte Anwendung der administrativen Autorität« eintritt, und zwar: Sie wird »eine Art Abstraktion des Zwanges zur Ordnung schlechthin « (Luhmann 1980, 257).

9 Luhmann (1990d, 116) spricht in diesem Fall von »Fernsynchronisation«.

10 Vgl. Luhmann 1984, 426: Man braucht Kalender, »aber nicht mehr, um wissen zu können, was zu bestimmten Zeitpunkten zu tun ist, sondern um verabreden zu können, was zu bestimmten Zeitpunkten zu tun ist« (Hervorh. i.O.). 
einer Episode annehmen können (Luhmann 1990c, 336). Diese für das Erfüllen von sozialen Funktionen entscheidende Zerlegbarkeit der Zeit in Episoden bzw. Perioden stimmt auch mit der Tatsache überein, dass die funktionale Differenzierung vor allem eine Zergliederung von teilsystemischen Eigenzeiten erzeugt (Luhmann 1997, 768; Nassehi 1993, 327), die sich in der Form einer ausdifferenzierten Episodisierung bzw. Periodisierung (d.h. in Form einer zeitlichen Polykontexturalität) der Gesellschaft entfaltet. Jedes Teilsystem verfügt nun über eine Eigendynamik, die gleichgültig gegenüber den Eigenzeiten anderer Teilsysteme sein kann, während eine allgemeine Koordinierung immer nur der persönlichen Initiative überlassen wird (man eilt aus dem Büro, um rechtzeitig einzukaufen). Die Termine des Rechtssystems stimmen also mit den Terminen der Politik nicht überein, aber die Verkündung eines Urteils zum juristisch richtigen Zeitpunkt (»just in time«) kann die Politik destabilisieren. Im Zusammenhang damit kann man vermuten, dass das Funktionieren formaler Organisationen unentbehrlich ist, welche die Behandlung dieser Logistik der Zeit planen, überwachen und sicherstellen.

Auf der Ebene der Interaktionen wird dieses auf Organisationen angewiesene Potenzial in eine Entkopplung von Interaktionszusammenhängen umgewandelt, welche paradoxerweise eine Steigerung des Formeneinprägungsvermögens von Interaktionen in der Gesamtgesellschaft fördert. Formale Organisationen (1) motivieren Individuen, an den Episoden teilzunehmen, indem sie ihnen Stellen und Geld anbieten; (2) sie gewährleisten die Verfügbarkeit über Interaktionen in dem Sinne, dass sie die Erwartung sicherstellen, an bestimmten Tagen und zu bestimmten Stunden Leute zu finden, die sich für Interaktionen zur Verfügung stellen (Kieserling 1999, 361); (3) sie ermöglichen es, eine große Menge von Interaktionen nebeneinander ablaufen zu lassen und aufeinander abzustimmen.

Das Formeneinprägungsvermögen kann ferner hohe Unwahrscheinlichkeit erreichen, wie zum Bespiel in Krankenhäusern, wo die Episodenbildung auf die Fähigkeit angewiesen ist, über Routinisierungen und rigide Zeitplanung (!) die Paradoxie derVoraussehbarkeit des Unvoraussehbaren zu behandeln. ${ }^{11}$ Die Organisationen tragen schließlich dazu bei, die öffentliche Zeit (die Zeit der öffentlichen Zugänglichkeit) von der privaten Zeit zu unterscheiden, und grenzen somit deutlich die Interaktionen (welche dennoch rational im temporalen Sinn organisiert werden (müssen), wie z.B. der Urlaub) von den Episoden ab - genau das, was in den mittelalterlichen Korporationen nicht möglich war, da die Person einer »Vollinklusion« aufgrund von meist attributiven Kriterien untergeordnet und die allgemeine soziale Zeit vielmehr in eine liturgische und eine alltägliche Zeit gespalten war. ${ }^{12}$

11 Ein funktionales Äquivalent könnten die Zeitungsredaktionen im System der Massenmedien sein.

12 Wendorff 1988, 165; Zerubavel 1985, 211ff. und insbes. 221, wo er über die »Bürokratisierung der beruflichen Verpflichtungen« spricht. Über den Mangel an einer Unterscheidung öffentlich / privat in den mittelalterlichen Korporationen vgl. auch Kieser 1989, insbes. 540. 
VI.

Unsere These besagt also, dass eine Episodisierung der Gesellschaft im eigentlichen Sinne nur dann entsteht, wenn Interaktion und Gesellschaft sich voneinander so stark differenzieren, dass man nicht mehr voraussetzen kann, dass die Gesellschaft unmittelbar auf Interaktionen angewiesen ist. Eben deshalb muss man von nun an Interaktionen organisieren.

Der enge Zusammenhang zwischen der Umstrukturierung sozialer Systeme und den allgemeinen Zeiteinrichtungen der Gesamtgesellschaft spiegelt sich vor allem in der Semantik deutlich wider. Die in der Neuzeit zunehmende Distanzierung von Interaktion und Gesellschaft und der hieraus herrührende Synchronisationsbedarf machen den Unterschied zwischen einer linearen homogenen Universalzeit, die zum Koordinieren von funktional ausdifferenzierten Episodisierungen bzw. Periodisierungen dient, und der nicht vergleichbaren Zeit des individuellen Erlebnisses erst sichtbar - und eben deshalb problematisch (Luckmann 1991, 160; McLuhan 1999, 155ff.). Diese Trennung zwischen Zeit des Bewusstseins und Zeit der Gesellschaft beweist nun deutlich, dass der Mensch außerhalb der Gesellschaft steht und eben dies ist in vielfältiger Weise emotional geladen, etwa durch Angst und Melancholie.

Aus diesem Zustand geht eine ganze Reihe von Zeitunterscheidungen hervor, die aber gerade infolge ihres entsprechenden Mangels an Reflexivität häufig eben das dahinter liegende Problem der gesellschaftlichen Umstrukturierung verstecken. Man unterscheidet also eine objektive Zeit, die normalerweise als quantitativ messbar und infolgedessen als anonym und »auf einen Warenwert reduzierbar « begriffen wird, von einer subjektiven Zeit, die im Gegenteil als qualitativ und persönlich erlebbar aufgefasst wird. Man fragt sich allerdings nicht, ob dieselbe Unterscheidung quantitativ/qualitativ bzw. objektiv/subjektiv eine qualitative oder quantitative bzw. objektive oder subjektive Unterscheidung sei (vgl. z.B. Heidegger 1916; Lukács 1991, 116ff.; Simmel 1957). Stattdessen könnte man die Hypothese untersuchen, nach der diese immer negativ bewertete Anonymisierung der sozialen Zeit eine Folge der Durchsetzung formaler Organisationen in der Neuzeit ist.

Durch Organisationen wird die Zeit in der Form von befristeten Tätigkeiten so strikt gekoppelt, dass die übrig bleibende Zeit als Freizeit (als Privatzeit) ausdifferenziert werden kann, welche ihrerseits eher zu befristeten programmierten Tätigkeiten neigt. Das Befristete generiert außerdem ein Knappheitsbewusstsein der Zeit (Luhmann 1971), das das Entstehen eines Spielraums alternativer Möglichkeiten fördert, die in Hinblick auf das Kombinieren weiterer Formen gleicher Art strikt gekoppelt werden können. Die Bedingungen der Episodenverknüpfung werden definitiv internalisiert, während dasselbe Episodenverknüpfen selbstreferentiell betrieben wird. Man schreibt sich an der Universität ein und beginnt somit eine Periode der Erziehung, welche in Episoden (Vorlesungen) und Termine (z.B. der Abgabetermin für eine schriftliche Arbeit) 
unterteilt wird. Aus diesem Grund wird wahrscheinlich allein für Arbeitslosen, die keine vordringlichen Termine haben, die Zeitgestaltung zu einem Problem (Heinemann/Ludes 1978, 229f.). Die Frage, ob und in welchem Maße, die durch die neuen telematischen Kommunikationsmedien gekennzeichnete Gesellschaft auf Episoden verzichten kann, bleibt hingegen offen.

\section{Literatur}

Cazeneuve, Jean (1996): Sociologia del rito. Ital. Übers. Milano: Il Saggiatore.

Cipolla, Carlo (1981): Le macchine del tempo. L'orologio e la società 1300-1700. Bologna: Il Mulino.

Condorcet, Marie-Jean-Antoine (1989): Quadro storico dei progressi dello spirito umano [1795]. Ital. Übers. Milano: BUR.

Dohrn-van Rossum, Gerhard (1989). Zeit der Kirche - Zeit der Händler - Zeit der Städte. Die mechanische Uhr und der Wandel des Zeitbewusstseins im Spätmittelalter. S. 89-119 in: Rainer Zoll (Hg.), Zerstörung und Wiederaneignung von Zeit. Frankfurt a.M.: Suhrkamp.

Eliade, Mircea (1984): Il sacro e il profano. Ital. Übers. Torino: Bollati Boringhieri.

Giddens, Anthony (1990): La costituzione della società. Ital. Übers. Milano: Edizioni di Comunità.

Havelock, Eric (1986): The Muse Learns to Write: Reflections on Orality and Literacy from Antiquity to the Present. New Haven/London: Yale U.P.

Heidegger, Martin (1916): Der Zeitbegriff in der Geschichtswissenschaft. Zeitschrift für Philosophie und philosophische Kritik 161, 173-188.

Heinemann, Klaus/Ludes, Peter (1978): Zeitbewusstsein und Kontrolle der Zeit. Kölner Zeitschrift für Soziologie und Sozialpsychologie 20, 220-243.

Kieser, Alfred (1989): Organizational, Institutional and Societal Evolution: Medieval Craft Guilds and the Genesis of Formal Organizations. Administrative Science Quarterly 34, 540-564.

Kieserling, André (1999): Kommunikation unter Anwesenden: Studien über Interaktionssysteme. Frankfurt a. M.: Suhrkamp.

Leclercq, Jean (1974): Zeiterfahrung und Zeitbegriff im Spätmittelalter. S. 1-20 in: Albert Zimmermann (Hg.), Antiqui und Moderni. Traditionsbewusstsein im späten Mittelalter. Berlin / New York: Walter de Gruyter.

Luckmann, Thomas (1991): The Constitution of Human Life in Time. S. 151-166 in: John Bender/David Wellbery (Hg.), Chronotypes. The Construction of Time. Stanford (Cal.): Stanford University Press.

Luhmann, Niklas (1971): Die Knappheit der Zeit und die Vordringlichkeit des Befristeten. S. 143-164 in: Ders., Politische Planung. Opladen. Westdt.Verlag.

Luhmann, Niklas (1980): Temporalisierung von Komplexität: Zur Semantik neuzeitlicher Zeitbegriffe. S. 235-300 in: Ders., Gesellschaftsstruktur und Semantik. Studien zur Wissenssoziologie der Moderne, Bd. 1. Frankfurt a.M.: Suhrkamp.

Luhmann, Niklas (1984): Soziale Systeme. Grundriß einer allgemeinen Theorie. Frankfurt a.M.: Suhrkamp.

Luhmann, Niklas (1987): The Evolutionary Differentiation between Society and Interaction. S. 112-131 in: Jeffrey Alexander (Hg.), The Micro-Macro Link. Berkeley/Los Angeles: The University of California Press.

Luhmann, Niklas (1990a): Anfang und Ende: Probleme einer Unterscheidung. S. 11-23 in: Niklas Luhmann/Karl Eberhard Schorr (Hg.), Zwischen Anfang und Ende: Fragen an die Pädagogik. Frankfurt a.M.: Suhrkamp. 
Luhmann, Niklas (1990b): Die Homogenisierung des Anfangs: Zur Ausdifferenzierung der Schulerziehung. S. 73-111 in: Niklas Luhmann/Karl Eberhard Schorr (Hg.), Zwischen Anfang und Ende: Fragen an die Pädagogik. Frankfurt a.M.: Suhrkamp.

Luhmann, Niklas (1990c): Die Wissenschaft der Gesellschaft. Frankfurt a.M.: Suhrkamp.

Luhmann, Niklas (1990d): Gleichzeitigkeit und Synchronisation. S. 95-125 in: Ders., Soziologische Aufklärung 5: Konstruktivistische Perspektiven. Wiesbaden:VS.

Luhmann, Niklas (1997): Die Gesellschaft der Gesellschaft. Frankfurt a. M.: Suhrkamp.

Lukács, György (1991): Storia e coscienza di classe [1922]. Ital. Übers. Milano: Edizioni SugarCo.

Mbiti, John (1969): African Religions and Philosophy. London/Nairobi: Heinemann.

McLuhan, Marshall (1999): Gli strumenti del comunicare [1964]. Ital. Übers. Milano: Il Saggiatore.

Mumford, Lewis (2005): Tecnica e cultura [1963]. Milano: Il Saggiatore.

Nassehi, Armin (1993): Die Zeit der Gesellschaft: Auf dem Weg zu einer soziologischen Theorie der Zeit. Opladen: Westdt. Verlag.

Schorr, Karl Eberhard (1990): Erziehung als Periode: Über die Organisation von Anfang und Ende. S. 112-132 in: Niklas Luhmann / Karl Eberhard Schorr (Hg.), Zwischen Anfang und Ende: Fragen an die Pädagogik. Frankfurt a.M.: Suhrkamp.

Simmel, Georg (1957): Die Großstädte und das Geistesleben. S. 227-242 in: Ders., Brücke und Tür. Stuttgart: KoehlerVerlag.

Sorokin, Pitirim / Merton, Robert (1937): Social Time: A Methodological and Functional Analysis. The American Journal of Sociology 42, 615-629.

Spencer Brown, George (1979): Laws of Form. New York: E.P. Dutton.

Teubner, Gunther (1987): Episodenverknüpfung: Zur Steigerung von Selbstreferenz im Recht. S. 423-446 in: Dirk Baecker et al. (Hg.), Theorie als Passion. Frankfurt a. M.: Suhrkamp.

Wendorff, Rudolf (1988), Der Mensch und die Zeit: Ein Essay. Opladen: Westdt.Verlag.

Zerubavel, Eviatar (1985): Ritmi nascosti. Orari e calendari nella vita sociale. Ital. Übers. Bologna: Il Mulino.

Dr. Alberto Cevolini

via Camatta 6

I-41100 Modena

alberto.cevolini@unimore.it 